\title{
Extracorporeal Membrane Oxygenation for Refractory Air Leak in a Child Presenting With Bacterial Tracheitis
}

\author{
Anna Dolgner MD, Jesse Bain DO, Stacey L Peterson-Carmichael MD, David A Turner MD, \\ and Kyle J Rehder MD
}

\section{Introduction}

In developed countries, bacterial tracheitis is an uncommon cause of acute upper airway obstruction in children, but the majority (57-91\%) of patients who present with this illness require invasive mechanical ventilation. ${ }^{1-3} \mathrm{Bac}-$ terial tracheitis is also associated with numerous complications, including ARDS, air leak syndromes, septic shock, and multiple organ dysfunction. ${ }^{1-3}$ We describe the first reported case of bacterial tracheitis in a young child complicated by bilateral pneumothoraces and pneumomediastinum that was managed with extracorporeal membrane oxygenation (ECMO).

\section{Case Summary}

A previously healthy 16-month-old female presented to our hospital's emergency department (Duke University Medical Center, Durham, North Carolina) with $4 \mathrm{~d}$ of rhinorrhea, worsening cough, and $2 \mathrm{~d}$ of increased work of breathing. On initial examination, she was noted to be febrile to $38.2^{\circ} \mathrm{C}$ with inspiratory stridor that was audible only with agitation. She was diagnosed with viral croup and given $0.6 \mathrm{mg} / \mathrm{kg}$ intramuscular dexamethasone and one dose of inhaled racemic epinephrine, and she

Dr Dolgner is affiliated with the Departments of Pediatrics and Internal Medicine, Duke University Medical Center, Durham, North Carolina. Dr Bain is affiliated with the Division of Pediatric Critical Care Medicine, Department of Pediatrics, Children's Hospital of Richmond, Richmond, Virginia. Drs Peterson-Carmichael, Turner, and Rehder are affiliated with the Division of Pediatric Critical Care Medicine, and Dr Peterson-Carmichael is also affiliated with the Division of Pediatric Pulmonary and Sleep Medicine, Department of Pediatrics, Duke Children's Hospital, Durham, North Carolina.

The authors have disclosed no conflicts of interest.

Correspondence: Kyle J Rehder MD, Division of Pediatric Critical Care, DUMC, Box 3046, Durham, NC 27710. E-mail: kyle.rehder@dm. duke.edu.

DOI: $10.4187 /$ respcare.03129 was admitted to the general pediatrics ward for continued observation.

Over the next several hours, her condition deteriorated as she developed retractions, tachypnea, and intermittent oxygen desaturations. This clinical change prompted transfer to the pediatric ICU for further management. A chest radiograph at the time of transfer demonstrated clear lung fields and a prominence of the upper airway described as overdistention of the hypopharynx, consistent with viral croup.

After transfer to the pediatric ICU, the patient's respiratory status continued to decline, and her increasing agitation interfered with the application of supplemental oxygen and heliox (helium-oxygen mixture). A dexmedetomidine infusion was initiated to facilitate placement of a face mask for delivery of her respiratory therapies, but she continued with worsening respiratory distress, lethargy, and significant hypoxemia. Given her rapid decompensation, the medical team performed an urgent and uncomplicated intubation, noting a grade 1 view of the vocal cords with moderate edema and thick secretions. Vancomycin and piperacillin/tazobactam were started for possible systemic bacterial infection.

Following intubation, the patient was placed on conventional ventilation, with settings of 24 breaths/min, peak inspiratory pressure of $24 \mathrm{~cm} \mathrm{H}_{2} \mathrm{O}$, PEEP of $6 \mathrm{~cm} \mathrm{H}_{2} \mathrm{O}$, and $\mathrm{F}_{\mathrm{IO}_{2}}$ of 0.6. Approximately 30 min later, she became acutely difficult to ventilate, requiring peak inspiratory pressures in excess of $40 \mathrm{~cm} \mathrm{H}_{2} \mathrm{O}$ to achieve tidal volumes of $3-4 \mathrm{~mL} / \mathrm{kg}$. A chest radiograph revealed bilateral basilar pneumothoraces, pneumomediastinum, and subcutaneous emphysema, which were not present on her immediate post-intubation film (Fig. 1). Bilateral chest tubes were placed to evacuate the pneumothoraces. Her first arterial blood gas post-intubation revealed respiratory acidosis, with $\mathrm{pH} 7.17, \mathrm{P}_{\mathrm{aCO}_{2}}$ of $71 \mathrm{~mm} \mathrm{Hg}, \mathrm{P}_{\mathrm{aO}_{2}}$ of $184 \mathrm{~mm} \mathrm{Hg}$, and bicarbonate at $25 \mathrm{mEq} / \mathrm{L}$. A trial of high-frequency jet ventilation was initiated to augment ventilation in an attempt to minimize ongoing air leak, but the patient had no discernible chest-wall movement despite a peak inspiratory pressure of $50 \mathrm{~cm} \mathrm{H}_{2} \mathrm{O}$ and continued air-trapping 


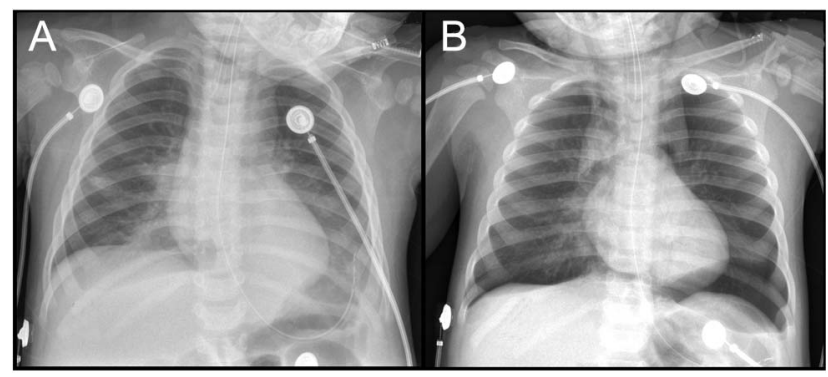

Fig. 1. A: The immediate post-intubation chest radiograph. B: The chest radiograph 30 min later, demonstrating bilateral pneumothorax and pneumomediastinum.

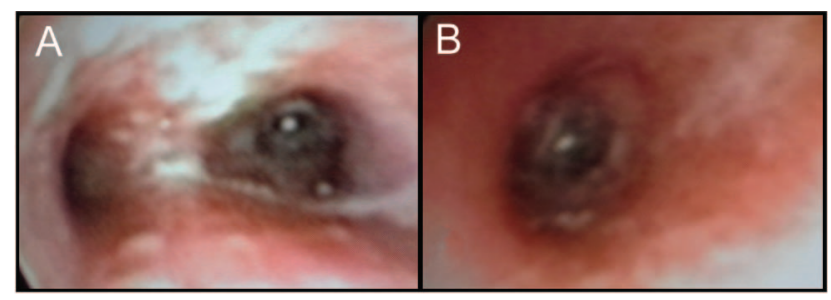

Fig. 2. Flexible bronchoscopy images of the carina $(A)$ and left main bronchus $(B)$, demonstrating extremely friable, edematous mucosa with purulent exudate adherent to the mucosal walls.

even at low rates (260-300 breaths/min). She was then transitioned to high-frequency oscillatory ventilation, with improved oxygenation at a mean airway pressure of $28 \mathrm{~cm} \mathrm{H}_{2} \mathrm{O}$, but again with poor chest-wall movement and inadequate ventilation despite maximal power (amplitude of $84 \mathrm{~cm} \mathrm{H}_{2} \mathrm{O}$ at a frequency of $8 \mathrm{~Hz}$ ). Arterial blood gas showed pH 6.98, $\mathrm{P}_{\mathrm{aCO}_{2}}$ of $105 \mathrm{~mm} \mathrm{Hg}, \mathrm{P}_{\mathrm{aO}_{2}}$ of $154 \mathrm{~mm} \mathrm{Hg}$, and bicarbonate at $24 \mathrm{mEq} / \mathrm{L}$. Due to continued inability to effectively ventilate, the decision was made to cannulate the patient for ECMO.

The unexpectedly small diameter of the internal jugular vein prevented the patient from being placed on venovenous ECMO via a double-lumen catheter; thus, venoarterial ECMO was initiated via the right internal jugular vein and right common carotid artery, with flows between 100 and $125 \mathrm{~mL} / \mathrm{kg} / \mathrm{h}$. To further assess the etiology for her rapid respiratory decompensation, flexible bronchoscopy was performed via her endotracheal tube, revealing friable, erythematous, edematous mucosa throughout the tracheobronchial tree with adherent purulent secretions consistent with bacterial tracheitis (Fig. 2). Bronchoalveolar lavage revealed parainfluenza virus type 2 and methicillinsusceptible Staphylococcus aureus. Serial bronchoscopies (performed 5 and $7 \mathrm{~d}$ after her initial bronchoscopy) revealed a progressive decrease in her secretions and healing mucosa along the tracheobronchial tree. The patient was treated with $14 \mathrm{~d}$ of nafcillin coupled with $8 \mathrm{~d}$ of gentamycin for antibiotic synergy at the recommendation of the infectious disease team.
The patient's initial ECMO course was complicated by superficial bleeding from the chest-tube sites. She also had an enlarging right pleural effusion, and on day 8 of ECMO, she developed cardiac tamponade from what proved to be a right-sided hemohydrothorax of unclear etiology. This required surgical exploration and placement of 2 chest tubes. Heparin infusion was held for $2 \mathrm{~h}$ before and $2 \mathrm{~h}$ after chest-tube placement and then resumed with a $10 \%$ reduction in activated clotting time target range, with minimal serosanguinous chest-tube output for the remainder of the ECMO course. The patient was successfully decannulated from ECMO after $10 \mathrm{~d}$. Chest tubes were removed, and the patient was successfully extubated on hospital day 17. She was ultimately discharged home on hospital day 35 without oxygen requirement or neurological deficits.

\section{Discussion}

This case illustrates the rapidity with which a child with bacterial tracheitis can decompensate and describes the novel use of ECMO as a rescue therapy for associated air leak. Bacterial tracheitis is an uncommon condition, with an estimated annual incidence of 0.1 case $/ 100,000$ children. ${ }^{3}$ Recognition of this serious disorder may be difficult, as many of the symptoms overlap with the much more common diagnosis of viral laryngotracheobronchitis (croup). As with our patient, the majority of children with bacterial tracheitis present with a viral prodrome, followed several days later by worsening stridor and increased work of breathing, likely representing bacterial superinfection after a viral illness. The minority of children present in a more fulminant manner, with high fever, toxic appearance, and rapid progression to respiratory failure. Children with bacterial tracheitis will generally appear more toxic than children with croup and will be less likely to respond to conventional therapies such as racemic epinephrine..$^{2,4,5}$ Common presenting symptoms of bacterial tracheitis include inspiratory or expiratory stridor (85\%), fever (89\%), and cough $(94 \%){ }^{2}$ Laboratory evaluation may reveal either leukocytosis or leukopenia, but bandemia is common. ${ }^{2,4,5}$ Lateral neck radiographs may demonstrate ragged tracheal contours, but these will not be present in all cases. ${ }^{6}$ Direct visualization by bronchoscopy is the definitive diagnostic test for bacterial tracheitis and will demonstrate erythematous, swollen subglottic tissues with thick, mucopurulent exudate. ${ }^{2,4}$ Bacterial tracheitis appears to be most common among infants and toddlers ( $0-6 \mathrm{y}$ of age), but has been reported in all age groups. Consistent with its frequent post-viral etiology, the peak incidence correlates with winter viruses such as influenza, parainfluenza, and respiratory syncytial virus.

Bacterial tracheitis has been associated with multiple serious complications, including hypotension (2\%), pneumothorax (1.3\%), and ARDS (1\%). ${ }^{3}$ Delayed recognition 


\section{ECMO FOR Refractory Air LEAK IN Bacterial Tracheitis}

can lead to difficult airway management and cardiac arrest. Historically, mortality for bacterial tracheitis was as high as $40 \%$, but currently, most children will make a full recovery, with a $3 \%$ estimated mortality rate. ${ }^{3}$ Volutrauma, barotrauma, and air leak are substantial risks given the airway pressures necessary to overcome lower airway obstruction from tenacious secretions, edematous airways, and sloughing mucosa, leading to possible air-trapping caused by a ball-valve mechanism.

The stretch imposed by conventional ventilator breaths during positive-pressure ventilation often causes repeat injury and can worsen air leak into interstitial and pleural spaces. In delivering tidal volumes smaller than the anatomic dead space, high-frequency ventilation causes negligible alveolar stretch and may halt progression of disease in air leak syndromes. ${ }^{7,8}$ However, some patients will have persistent inadequate gas exchange due to poor lung compliance and high airway resistance secondary to interstitial air accumulation.

When air leak is progressive, as was the case for this patient, ECMO may be necessary. Although this is the second reported case of ECMO use for bacterial tracheitis, ${ }^{9}$ it is the first case of a patient being supported with ECMO for bacterial tracheitis complicated by air leak. In air leak syndromes, use of extracorporeal lung support facilitates dramatic reduction in ventilator settings, allowing lung rest and healing. ${ }^{10}$ Over 4,000 ECMO cases reported to the Extracorporeal Life Support Organization registry have reported an air leak diagnosis (pneumothorax or emphysema) ${ }^{11}$; however, only 133 list air leak as the primary indication for ECMO. ${ }^{11}$ Of these cases, the overall survival was $70 \%$ and similar across age groups (81/118 [69\%] for neonates and 12/15 [80\%] for pediatric cases). The majority $(73 \%)$ of these patients were supported with venoarterial ECMO, with a trend toward increased venovenous ECMO use in recent years. The median duration of ECMO for survivors was $131 \mathrm{~h}$ (range of 8-690 h) and was significantly longer in pediatric cases than in neonatal cases ( $227 \mathrm{~h}$ vs $126 \mathrm{~h}, P=.003$ ). There was a peak of 13 ECMO cases for air leak in children in 1996 and 5 cases in 2012. ${ }^{11}$

In conclusion, bacterial tracheitis is an uncommon but potentially fatal cause of upper airway obstruction in young children. Early recognition can be challenging, as it mimics the symptoms of common croup, but is of utmost importance, as this is key for minimizing complications. In the most severe cases, ECMO may be required to allow adequate gas exchange while promoting lung rest and recovery.

\section{Teaching Points}

- Bacterial tracheitis is an important diagnostic consideration in children presenting with presumed viral croup.

- Conventional ventilation in patients with bacterial tracheitis can result in pneumothorax due to the friability of the inflamed airways and the tenacity of the tracheal secretions.

- High-frequency ventilation may be beneficial for patients with air leak syndromes, but the most severely affected patients may require extracorporeal support.

- ECMO provides respiratory support while allowing the tracheal and bronchial mucosa to recover by avoiding high-pressure mechanical ventilation in severe cases of bacterial tracheitis.

\section{REFERENCES}

1. Bernstein T, Brilli R, Jacobs B. Is bacterial tracheitis changing? A 14-month experience in a pediatric intensive care unit. Clin Infect Dis 1998;27(3):458-462.

2. Hopkins A, Lahiri T, Salerno R, Heath B. Changing epidemiology of life-threatening upper airway infections: the reemergence of bacterial tracheitis. Pediatrics 2006;118(4):1418-1421.

3. Tebruegge M, Pantazidou A, Thorburn K, Riordan A, Round J, De Munter C, et al. Bacterial tracheitis: a multi-centre perspective. Scand J Infect Dis 2009;41(8):548-557.

4. Al-Mutairi B, Kirk V. Bacterial tracheitis in children: approach to diagnosis and treatment. Paediatr Child Health 2004;9(1):25-30.

5. Rotta AT, Wiryawan B. Respiratory emergencies in children. Respir Care 2003;48(3):248-258; discussion 258-260.

6. Bjornson CL, Johnson DW. Croup. Lancet 2008;371(9609):329-339.

7. Ellsbury DL, Klein JM, Segar JL. Optimization of high-frequency oscillatory ventilation for the treatment of experimental pneumothorax. Crit Care Med 2002;30(5):1131-1135.

8. Shen HN, Lu FL, Wu HD, Yu CJ, Yang PC. Management of tension pneumatocele with high-frequency oscillatory ventilation. Chest 2002;121(1):284-286.

9. Carrel T, Gygax E, Jenni HJ, Wagner B. Successful extracorporeal life support using a new micro-diagonal pump in a child with acute laryngotracheobronchitis, lung failure, and untractable septic shock. J Thorac Cardiovasc Surg 2007;133(3):824-825.

10. Turner DA, Ofori-Amanfo G, Williford WL, Cheifetz IM. Lung protective ventilation: a summary of the current evidence from the 2012 American Association for Respiratory Care International Congress. Expert Rev Respir Med 2013;7(3):209-212.

11. Miyoshi K, Oto T, Okazaki M, Yamane M, Toyooka S, Goto K, et al. Extracorporeal membrane oxygenation bridging to living-donor lobar lung transplantation. Ann Thorac Surg 2009;88(5):e56-357. 